\title{
Microchannels in conventional single-mode fibers
}

\author{
Y. Lai, K. Zhou, L. Zhang, and I. Bennion \\ Photonics Research Group, School of Engineering and Applied Science, Aston University, Birmingham B4 7ET, UK \\ Received May 17, 2006; accepted June 8, 2006; \\ posted June 14, 2006 (Doc. ID 71077); published August 9, 2006 \\ Microchannels are fabricated into conventional single-mode fibers by femtosecond laser processing and \\ chemical etching. Fabrication limitations imposed by the fiber geometry are highlighted and resolved \\ through a simple technique without compromising fabrication flexibility. A microfluidic fiber device consist- \\ ing of a $4 \mu \mathrm{m}$ wide microchannel that intersects the fiber core for refractive index sensing is further \\ demonstrated. (C) 2006 Optical Society of America \\ OCIS codes: $220.4000,060.2310,290.3030$.
}

The advancement toward microphotonics components for modern biomedical, chemistry, and sensing applications has generated increasing interest in microfluidic optical devices. Within the broad-ranging development of optical microfluidics, there has been interest to integrate such advances into fiber optics to incorporate the operational merits of optical fibers. To date, such developments have adopted special photonic crystal fibers where the fluids are accessed within the cladding air holes. ${ }^{1}$ Alternative reported methods include immersing cladding-etched fibers or fiber gratings into planar bulk-machined fluid channels. ${ }^{2}$ In general, the operations of these proposed device formats are based on the interactions between the evanescent field and the surrounding fluids, which modify the optical waveguiding properties of the fiber.

The foundation of microfluidic fiber devices anchors on an effective overlap between the optical field and the channel fluids. For a guided light wave, its interaction with the fluid can be maximized when both the light and the fluid are confined simultaneously in a waveguide core. Such assembly is desirable for achieving a large dynamic response range and short interaction length. To date, practical implementations to realize such microfluidic configurations have relied on abutting two fiber surfaces to the microfluidic channel, ${ }^{3}$ while other proposed techniques involve coupling fibers into an optical hollowcore planar waveguide.

Toward improved channel integration, reliability, and ease of production, an alternative concept is considered here where the microchannels are fabricated directly into an optical fiber. It is advantageous to do so since the desired alignment, and hence the interactions between the microfluidic channels and the optical waveguide, can be accurately defined and guaranteed. The fiber waveguide constitutes the backbone conduit for input/output light delivery, and effective light-fluid interaction is achieved when the microfluidic channel intersects the optical pathway. To the best of our knowledge, there has been no description to date of microfluidic fiber devices based on such engineered integrated microchannels.

In this Letter we report, for the first time to our knowledge, the fabrication of microchannels within single-mode fibers based on femtosecond laser exposure combined with chemical etching. The fabrication process takes advantage of the high spatial resolution inherent to femtosecond laser inscription, and the preferential chemical etching of the modified material to realize the designed microchannels within the fiber. ${ }^{5}$ We highlight the fabrication limitations imposed by the surface geometry of the fiber and demonstrate a simple solution involving an addition of a glass slip attachment. We further illustrate a microfluidic fiber device consisting of a microchannel that intersects the fiber core for refractive index sensing applications. The index measurements are achieved based on straightforward optical power detections, and the effective light-fluid interaction length of the microfluidic fiber device is only of the order of $4 \mu \mathrm{m}$.

The fabrication process involves two main steps: (1) inscription of the desired structure into the fiber by using a tightly focused femtosecond laser beam, and (2) etching of the fiber in a solution of $5 \%$ hydrofluoric acid (HF) for selective removal of the laser-modified regions. In the laser inscription process, the femtosecond laser pulses (center wavelength $=800 \mathrm{~nm}$ ) were focused into the silica fiber by using a $100 \times$ objective lens with a NA of 0.55 and a working distance of $13 \mathrm{~mm}$. The laser pulse width was measured to be $\sim 150 \mathrm{fs}$, and the repetition rate was at $1 \mathrm{kHz}$. The focused spot size was evaluated to be $\sim 1.5 \mu \mathrm{m}$, and the average pulse energy was measured to be $550 \mathrm{~nJ}$. The fiber was mounted on a dual-axes air-bearing translation stage, so that the desired structure could be written by moving the fiber with respect to the propagation direction of the laser beam.

The fiber was first translated at $10 \mu \mathrm{m} / \mathrm{s}$ along the direction of propagation of the laser beam so as to inscribe a straight-line track transversely through the fiber core. A single-pass exposure over a distance much larger than the fiber diameter was carried out to ensure the inscription process covered the entire fiber. A high-resolution optical microscope was then used to visually inspect the inscribed feature within the optical fiber.

As shown in Fig. 1a, the femtosecond laser-induced index modification in the fiber is clearly visible. More important, a distorted feature is obtained instead of the expected straight-line track. The inscription process was repeated whereby the fiber was translated in the reverse direction and a similar distorted laser- 


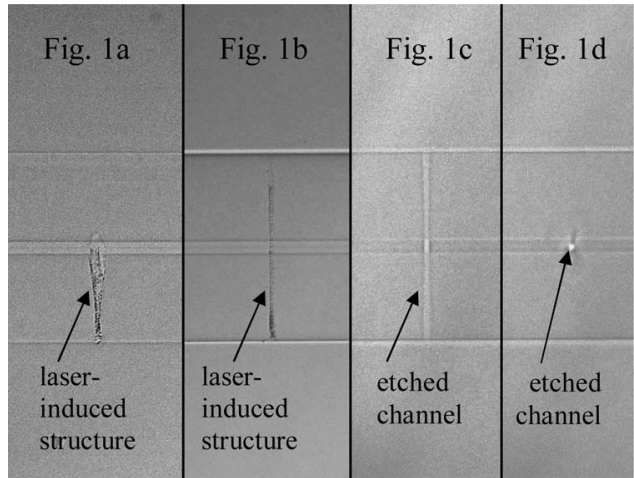

Fig. 1. a, Microscope image of the femtosecond laserinduced index modification in the fiber after direct laser exposure. $b$, Image of the inscribed straight-line track within the fiber when a glass slip attachment is adopted during the laser exposure. $c$ and $d$, Images of the etched channel in the fiber viewed at orthogonal angles, indicating that the microchannel intersects the fiber core.

induced structure was obtained. It is clear that the inscribed feature expands as it approaches the fiber core and subsequently fades away as the laser beam moves further into the fiber. This is attributed to the curved surface of the fiber, which defocuses the incident femtosecond laser beam, leading to a decrease in fluence. This effect increases with increasing processing depth into the fiber, and the fluence subsequently drops below the necessary threshold to cause any significant index modification.

To alleviate the defocusing-induced distortions, a simple approach was attempted whereby the fiber surface geometry, presented to the path of the incident femtosecond laser beam, is rectified by using a closely attached flat glass slip. Conceptually similar to the use of an oil-immersion lens, this approach however does not restrict fabrication flexibility and is easily implemented within the current fabrication setup. The inscription process was repeated under the same conditions except a conventional microscope glass slip (thickness $=125 \mu \mathrm{m}$ ) was attached to the fiber on the near side of the objective lens as shown schematically in Fig. 2. Index-matching oil was used to remove any air gap and to form a continuum between the fiber and the glass slip. The resultant inscribed feature in the fiber based on this technique is as shown in Fig. 1b, and an undistorted straight-line track through the fiber core was obtained expectedly.

After the laser inscription process, the fiber was chemically etched in the HF solution assisted by an ultrasonic bath at ambient conditions for $\sim 12 \mathrm{~min}$. The optimum etching time was determined through real-time monitoring of the optical loss through the fiber. The fiber was removed from the HF solution when the rate of transmission loss slowed down drastically; further etching only serves to widen the etched channel. The processed fiber was visually inspected under the microscope, and images of the etched feature within the fiber are as shown in Figs. 1c and 1d. Evidently, a microchannel that intersects the fiber core has been achieved.

Even though the detail morphology of the microchannel cannot be accurately determined through the microscope images, one can observe a direct spatial correlation between the laser-modified track and the region that is preferentially etched that led to the creation of the microchannel. However, the etched zone extends over a distance that is larger than the laser-modified track, highlighting the presence of laser-induced densification/stress zones around the inscribed region. ${ }^{5,6}$ Taking note that the etching rate of an identical, unexposed fiber under the same conditions was measured to be $\sim 0.06 \mu \mathrm{m} / \mathrm{min}$, the lasermodified feature here is therefore $>200$ times more susceptible to the etching process. This etching selectivity is useful for creating narrow channels as well as preserving the structural integrity around the inscribed feature. The physical dimensions of the microchannel were determined under a $40 \times$ oilimmersion microscopic lens as shown in Fig. 3. The width of the channel tapers very slightly toward the fiber core because the inside of the fiber experiences a shorter exposure time to the HF solution compared with regions nearer to the surfaces. ${ }^{5}$ The channel width, measured to be $4.0 \mu \mathrm{m}$, near to the fiber core is smooth and uniform but deteriorates noticeably near to the fiber surfaces. We believe this is due to the clipping effect of the laser beam whose power is perturbed as the beam transits the surface edge. The induced stress fields within the laser-modified regions are consequently disturbed, leading to a nonhomogeneous etched pattern. ${ }^{6}$ Such features can be further reduced if optimally matched index oil is used between the glass slip and the fiber.

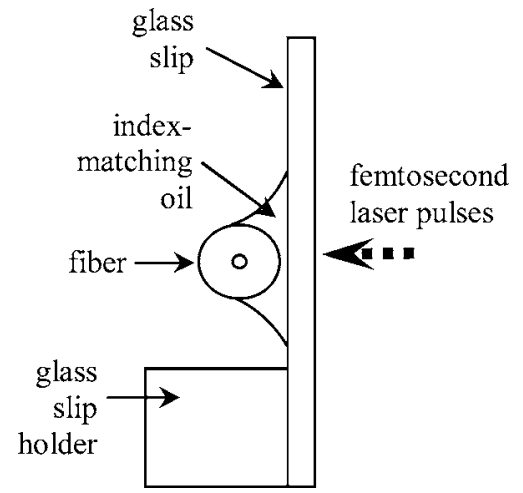

Fig. 2. Schematic setup of using a glass slip to alleviate the effect of the fiber curved surface. The diagram is not drawn to scale.

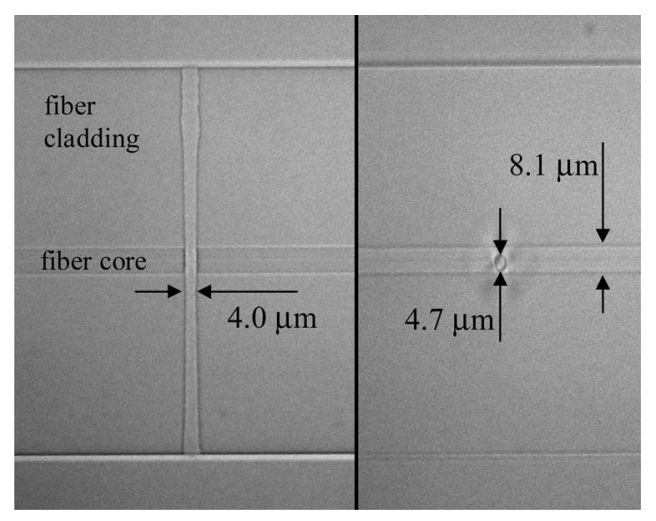

Fig. 3. Microscope images of the microchannel within the fiber viewed under a $40 \times$ oil-immersion microscopic lens. 


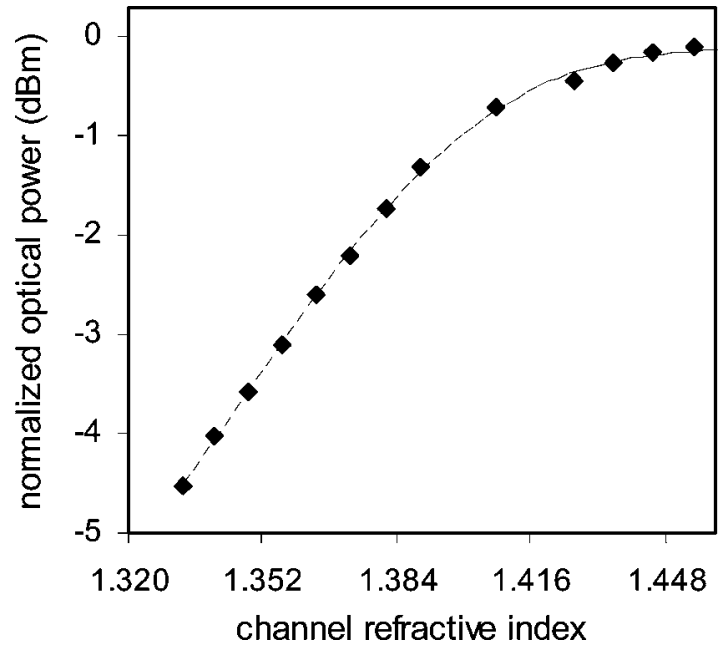

Fig. 4. Normalized transmitted optical power through the microfluidic fiber device at different microchannel refractive indices.

The presence of the microchannel within the fiber core introduces scattering loss to the propagating core mode. The refractive index of the channel on the other hand affects the amount of scattering, hence the transmission loss, through the fiber device. Taking into account the dimensions of the microchannel, the required fluid volume to fill the channel for optical probing is minimal. The effective light-fluid interaction length is essentially the diameter of the microchannel and is only on the order of $4 \mu \mathrm{m}$. A series of mixtures consisting of different volume concentrations of glycerine in water were prepared to create solutions of different indices ranging from 1.333 to 1.475 for the microchannel fill. The transmission power through the microfluidic fiber device was measured at different channel indices by using a C-band broadband source and an optical spectrum analyzer as the detection unit. The normalized transmitted power at $1538 \mathrm{~nm}$ through the microfluidic fiber device at different channel indices is plotted as shown in Fig. 4.

Between index values of 1.333 to 1.407 , the transmitted power through the microfluidic fiber device exhibits a linear increment with increasing microchannel index at a rate of $0.56 \mathrm{~dB} / 10^{-2}$. Further index increments up to 1.475 saw a much smaller improvement to the transmission loss, which eventually levels at $\sim 0.07 \mathrm{~dB}$. For an optical power detection resolution of $0.01 \mathrm{~dB}$, the resolution in the refractive index measurement with this simple device configuration is therefore on the order of $1.79 \times 10^{-4}$ between index values of 1.333 to 1.407. Evidently, the dynamic power response range, hence sensitivity, can be increased by cascading a larger number of microchannels. The spatial resolution of the femtosecond laser inscription process can allow high-density precisely positioned microfluidic channels within the fiber. It is also important to note that because of the miniature cross-sectional feature size of the microchannel, the polarization dependent loss of the fiber device is relatively insignificant and measured to be $<0.08 \mathrm{~dB}$. These attributes highlight the potential of such microfluidic fiber devices as ultrasmall indexsensing components or in-fiber attenuators for many photonics applications. Furthermore, with the inclusion of fiber grating structures, we believe a range of devices with enhanced functionalities and performances can be made possible.

In conclusion, the fabrication of microchannels within conventional single-mode fibers is demonstrated. Fabrication limitations imposed by the fiber geometry are highlighted and resolved by using a simple glass slip attachment. A microfluidic fiber device consisting of a $4 \mu \mathrm{m}$ wide microchannel for refractive index sensing applications is further demonstrated. The results obtained illustrate the potential attributes of engineered integrated microchannels in fibers for tunable photonics and sensing applications.

Y. Lai's e-mail address is laiy@aston.ac.uk.

\section{References}

1. J. B. Jensen, L. H. Pedersen, P. E. Hoiby, L. B. Nielsen, T. P. Hansen, J. R. Folkenberg, J. Riishede, D. Noordegraaf, K. Nielsen, A. Carlsen, and A. Bjarklev, Opt. Lett. 29, 1974 (2004).

2. J. Hsieh, P. Mach, F. Cattaneo, S. Yang, T. Krupenkine, K. Baldwin, and J. A. Rogers, IEEE Photon. Technol. Lett. 15, 81 (2003).

3. C. Grillet, P. Domachuk, V. Ta'eed, E. Mägi, J. A. Bolger, B. J. Eggleton, L. E. Rodd, and J. CooperWhite, Opt. Express 12, 5440 (2004).

4. S. Campopiano, R. Bernini, L. Zeni, and P. M. Sarro, Opt. Lett. 29, 1894 (2004).

5. A. Marcinkevicius, S. Juodkazis, M. Watanabe, M. Miwa, S. Matsuo, H. Misawa, and J. Nishii, Opt. Lett. 26, 277 (2001).

6. Y. Bellouard, A. Said, M. Dugan, and P. Bado, Opt. Express 12, 2120 (2004). 